\title{
Serum Response Factor Indirectly Regulates Type I Interferon-Signaling in Macrophages
}

\author{
Lan Xie, ${ }^{1-3}$ Amy L. Sullivan, ${ }^{2}$ Jana G. Collier, ${ }^{2}$ and Christopher K. Glass ${ }^{2}$
}

Serum response factor (SRF) is required for diverse aspects of development and homeostasis, but potential roles in the regulation of inflammation and immunity have not been systematically investigated. Here, we demonstrate that SRF is unexpectedly required for optimal responses of elicited peritoneal macrophages to type I interferons. Knockdown of SRF expression in these cells impairs induction of numerous interferon (IFN)stimulated genes (ISGs) in response to zymosan, LPS, and poly I:C. This effect is primarily due to a defect in the ability of induced type I interferons to mediate secondary activation of ISGs. SRF does not appear to be required for expression of established components of the type I interferon signaling pathway, with IFN- $\beta$-dependent phosphorylation of STAT1 and STAT2 normally occurring in SRF-depleted macrophages. Collectively, these findings suggest that SRF can indirectly modulate type I interferon-signaling, without interfering with the classic JAK/STAT/ISGF3 pathway.

\section{Introduction}

$\mathrm{R}$ APID ACTIVATION of the innate immune system provides the first line of defense against invading pathogens and initiates the development of acquired immunity. Macrophages play important roles in this response by virtue of their abilities to sense and phagocytose microorganisms and release numerous mediators that activate pro-inflammatory signaling pathways and recruit additional immune cells to sites of infection. To fulfill these functions, macrophages express a variety of pattern recognition receptors (PRRs) on their cell membranes that enable recognition of conserved microbial structures referred to as pathogen-associated molecular patterns. One key family of PRRs, Toll-like receptors (TLRs), consists of 13 members that recognize lipid, carbohydrate, peptide, and nucleic-acid components of different groups of microorganisms (Akira and Takeda 2004). For example, TLR2 recognizes bacterial surface di- and tri-acyl lipopeptides, TLR3 recognizes double-stranded RNA as a signature for viral infection, and TLR4 recognizes LPS, a major component of the gram-negative bacterial cell wall (Barton and Medzhitov 2003). Some TLRs collaborate with other receptors to generate a pro-inflammatory response. For example, dectin-1, a C-type lectin receptor recognizing fungal wall-derived $\beta$-glucans, has been shown to cooperate with TLR2 to mediate zymosan or fungal pathogen-induced pro-inflammatory responses (Yadav and Schorey 2006).
Upon ligand binding, TLRs regulate inflammatory responses through activation of downstream signaling cascades to activate numerous downstream transcription factors, including members of the NFKB, AP1, and interferonregulatory factor (IRF) transcription factor families (Goodridge and Harnett 2005). Upon receptor activation, NFkB, AP1, and IRF proteins are capable of inducing the expression of hundreds of pro-inflammatory genes that comprise the immediate phase of TLR signaling (Li and Verma 2002; Honda and Taniguchi 2006). Products of the primary response genes are involved in initiating secondary responses (Panne and others 2004). TLR induced production and secretion of soluble interferon- $\beta$ (IFN- $\beta$ ) binds to the interferon $\alpha / \beta$ receptor (IFNAR) and activates the JAK-STAT pathway, which results in the phosphorylation and translocation of interferon-stimulated gene factor 3 (ISGF3) and induction of interferon-stimulated genes (ISGs) that are important for antiviral host defense (Lehtonen and others 1997; Gale and Foy 2005).

Serum response factor (SRF) is a member of the MADS (Mcm1, Agamous, Deficiens, and SRF) family of eukaryotic transcription factors (Chai and Tarnawski 2002). Binding of SRF to DNA is mediated by a consensus cis-element known as a CArG box, with a sequence of $\mathrm{CC}(\mathrm{A} / \mathrm{T})_{6} \mathrm{GG}$ (Sun and others 2006). It is well known that SRF directly regulates the expression of transcription factors, such as $c$-fos, egr 1 , and Jun, which directly regulate the expression of genes that are

\footnotetext{
${ }^{1}$ Medical Systems Biology Research Center, School of Medicine, Tsinghua University, Beijing, China.

${ }^{2}$ Department of Cellular and Molecular Medicine, University of California, San Diego, La Jolla, California.

${ }^{3}$ National Engineering Research Center for Beijing Biochip Technology, Beijing, China.
} 
important for initiating growth and differentiation of a variety of cell types (Chai and Tarnawski 2002; Miano 2003; Treisman 1986). Another significant group of SRF target genes are those involved in the actin cytoskeletal system, such as cofilin, vinculin, and contractile genes (Miano 2003; Miano and others 2007). Genetic deletion of Srf revealed that these SRF-dependent gene expression programs are required for the proper growth, development, and function of tissues such as the heart, muscle, liver, brain, and skin (Schratt and others 2002; Miano and others 2004; Alberti and others 2005; Lahoute and others 2008; Sun and others 2009). However, the roles of SRF in regulating cellular functions in immunity are not well studied. Previous work indicates that under basal conditions, SRF regulates cytoskeletal gene expression in macrophages (Sullivan and others 2010). Here, our studies reveal an unexpected requirement for SRF in the regulation of secondary interferon responses to type I interferon induced by TLR signaling. This regulation was shown to occur through a noncanonical mechanism independent of the classical JAK-STAT-ISGF3 signal transduction pathway.

\section{Materials and Methods}

\section{Reagents and plasmids}

Zymosan A, LPS, polyI:C, IFN- $\gamma$, Actinomycin D (Act-D), and cycloheximide (CHX) were obtained from Sigma. Thioglycollate was from BD Biosciences. IFN- $\alpha$ and IFN- $\beta$ were obtained from PBL InterferonSource.

\section{Expression array profiling}

Total RNA (isolated by RNeasy kit; Qiagen) was prepared from untreated or zymosan treated $(1 \mathrm{mg} / \mathrm{mL}, 1$ or $6 \mathrm{~h}$ ) thioglycollate-elicited macrophages. About $0.5 \mu \mathrm{g}$ of purified RNA per sample was labeled using the LRILAK PLUS, 2 color Low RNA Input Linear Amplification kit and hybridized to an Agilent Whole Mouse Genome Microarray $4 \times 44 \mathrm{~K}$ 60 mer slides according to the manufacturer's instructions. Slides were scanned using the Agilent GZ505B Scanner and analyzed using Gene Spring Software (Agilent) or DAVID (http://david.abcc.ncifcrf.gov/home.jsp), the web-based functional-annotation tool for Gene Ontology Analysis.

\section{Cell culture and transient transfection}

Thioglycollate-elicited, peritoneal macrophages were prepared as previously described (Ghisletti and others 2007) from 6-8 week old, male, C57BL/ 6 mice (Harlan). For RNAi experiments in primary macrophages, 0.75 million cells were transfected with control or SMARTpool siRNAs (100 nM; Dharmacon) directed against Srf mRNA using the Deliver $\mathrm{X}$ transfection reagent (Panomics) according to the manufacturer's instructions. Cells were used for experiments $48 \mathrm{~h}$ post-transfection and target gene knockdown was validated by Q-PCR.

\section{RNA isolation and Q-PCR}

Total RNA (isolated by RNeasy kit; Qiagen) was prepared from primary macrophages. $1 \mu \mathrm{g}$ of total RNA was used for cDNA synthesis according to the manufacturer's instructions (Superscript III; Invitrogen), and $1 \mu \mathrm{L}$ of cDNA was used for Q-PCR analysis using SYBRGreenER master mix (Invitrogen) and gene-specific primers. Primer sequences are available upon request. Q-PCR analysis was performed on an Applied Biosystems 7300 Real-Time PCR system. Values were normalized to Gapdh expression (Livak and Schmittgen 2001). Briefly, for each sample, the target gene and the housekeeping gene Gapdh content were both examined by the Real-time PCR system and the CT values were recorded. The relative amount of the target gene was represented by the value normalized to Gapdh $\left(\Delta \mathrm{CT}=\mathrm{CT}_{\text {(Target gene) }}\right.$ $\mathrm{CT}_{(\text {Gapdh })}$, Expression level $\left.=2^{-\Delta \mathrm{CT}}\right)$.

\section{Western blotting}

For the whole cell lysate analysis, $2-3 \times 10^{6}$ primary macrophages were lysed with RIPA buffer $(40 \mathrm{mM}$ Tris, pH7.4, $150 \mathrm{mM} \mathrm{NaCl}, 1 \%$ Triton X-100, 0.2\% SDS, 0.5\% Nadeoxycholate, $1 \times$ complete protease inhibitors (Roche), $1 \mathrm{mM}$ PMSF, $5 \mu \mathrm{M}$ E64, and $5 \mu \mathrm{M}$ MG132). Nuclear and cytoplasmic extracts were prepared from thioglycollate-elicited macrophages using a commercial kit (NE-PER; Pierce). Western blotting was performed as described in detail by Impey and others (2002). Briefly, extracts were resolved by SDS-PAGE (4\%-12\% NuPAGE; Invitrogen), transferred to PVDF (Immobilon-P; Millipore) and immunoblotted using anti-STAT1 (Santa Cruz), anti-STAT2 (Santa Cruz), anti-pSTAT1 (Santa Cruz), anti-p-STAT2 (Upstate), anti-GAPDH (Abcam), and anti-lamin (Cell Signaling) antibodies. The appropriate alkaline phosphatase-conjugated secondary antibodies (Jackson) were then used and developed with the CDP-Star chemiluminescent reagent (Applied Biosystems) according to the manufacturer's instructions.

\section{De novo motif analysis}

Enriched sequence motifs in promoters of differentially expressed genes were identified as previously described (Heintzman and others 2009; Heinz and others 2010; Sullivan and others 2010). Briefly, peak sequences $( \pm 200 \mathrm{bp}$ from the center of the peak) were compared to 50,000 random, genomic sequences that were generated to match the size and GC-content of the peak sequences (to remove the sequence bias introduced by CpG islands). Motifs of 8,10 , and 12 base pairs were identified by screening all oligos for enrichment in the target set compared to the background set, with an allowance of $2 \mathrm{bp}$ mismatches to increase the sensitivity of the method. The top 200 oligonucleotides of each length with the lowest p-values were then converted into probability matrices and heuristically optimized to maximize hypergeometric enrichment of each motif in the given data set. As optimized motifs were found they were removed from the data set to facilitate the identification of additional motifs. Sequence logos were generated using WebLOGO (http://weblogo .berkeley.edu/).

\section{Results}

\section{SRF is globally required for ISG expression in macrophages}

To identify transcription factors not previously recognized to contribute to innate immune responses, we performed de novo motif analysis of promoters of genes induced in macrophages by zymosan, a ligand for the dectin- 1 receptor and TLR2/6 (Karumuthil-Melethil and others 2008). Inducible 
promoters were identified using Agilent Mouse Whole Genome Microarrays, using a confident, nonredundant subset of 20,721 probes that could be linked to specific transcriptional start sites (TSS). A total of 472 genes were identified as induced after $1 \mathrm{~h}$ of zymosan treatment (Supplementary Table S1-Part I; Supplementary Data are available online at www.liebertpub.com/jir) using an induction threshold of 2-fold and a false positive rate cutoff of 0.01 . These genes were involved in biological processes such as "response to wounding," "immune response," and "regulation of apoptosis," and so on by Gene Ontology analysis (Supplementary Table S1-Part II). De novo motif analysis was performed and motif enrichment was considered to be significant at a $P$-value of $<1 \mathrm{e}^{-10}$ compared to the frequency of the same motif in the set of promoters that were not induced. In evaluating promoters induced $1 \mathrm{~h}$ after zymosan treatment, binding sites for $\mathrm{NF \kappa B}$ were highly enriched, as expected (Goodridge and Harnett 2005). Unexpectedly, an SRF/MADS (CArG box) motif was the third most highly enriched motif in the zymosan-induced genes with a $P$-value of $7.839 \mathrm{e}^{-13}$ (Fig. 1A)

Based on this observation, we evaluated the impact of knocking down Srf mRNA levels on zymosan responses of elicited mouse peritoneal macrophages. Transfection with SRF-specific siRNAs reduced Srf mRNA levels to approximately $20 \%$ of the levels present in macrophages transfected with control siRNAs (Fig. 1B). Control and SRF siRNAtransfected cells were then treated with zymosan for 0,1 , or $6 \mathrm{~h}$ and total mRNA was subjected to transcriptome analysis using Agilent microarrays. To identify SRF-dependent genes, we filtered the set of transcripts that were reduced by more than 33\% with SRF-specific siRNAs. This resulted in the identification of 263, 370, and 436 genes that exhibited impaired induction upon SRF knockdown at 0,1 and $6 \mathrm{~h}$ of zymosan treatment, respectively. Since we focused our studies on the role of SRF in inflammatory responses, we defined SRF-dependent inflammatory response genes as those transcripts that were induced more than 3 -fold after $6 \mathrm{~h}$ of zymosan treatment and reduced by more than $33 \%$ in macrophages transfected with SRF-specific siRNAs at either 1 or $6 \mathrm{~h}$ of zymosan treatment. Filtering the data using these criteria resulted in the identification of 49 genes that exhibited impaired induction upon SRF knockdown at $1 \mathrm{~h}$ and an overlapping but distinct set of 64 genes exhibiting impaired knockdown at $6 \mathrm{~h}$ (Fig. 1C). Both sets of genes were significantly enriched for functional annotations linked to
A

\begin{tabular}{ccc} 
Motif enriched & Name & P-value \\
\hline GGAAATCCCC & REL/RELA & $4.7 \mathrm{E}-24$ \\
CTTATAGCCA & MEF2/MADS & $1.8 \mathrm{E}-13$ \\
CATATATGGA & SRF/MADS & $7.8 \mathrm{E}-13$ \\
CGGTGACGTC & bZIP/AP-1 & $5.2 \mathrm{E}-12$
\end{tabular}

D Category

response to external stimulus response to pest, pathogen or parasite sphingolipid metabolism immune response chemotaxis response to wounding

E

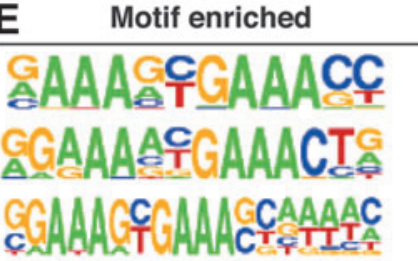

\section{P-value}

0.0008

0.0021

0.0027

0.0047

0.0056

0.0070

Name P-value

IRF1/TRP.

CLUSTER

STAT1/Stat

2.35E-19

IRF2/TRP CLUSTER
B

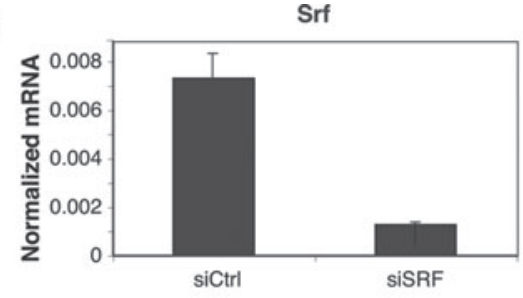

C

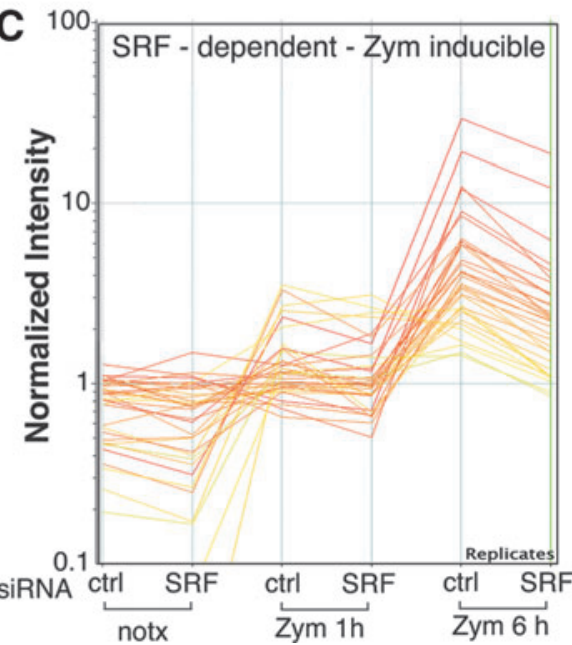

FIG. 1. Genome wide analysis of the role of serum response factor (SRF) in transcription regulation in macrophages. (A) Logos of sequence motifs over-represented in the promoters of genes induced $>2$-fold in elicited macrophages following zymosan treatment for $1 \mathrm{~h}$. Relative height of letters indicates the frequency of occurrence at each position. A binding site for SRF is the third most highly enriched sequence motif in the promoters of this set of genes. (B) Srf knockdown efficiency was confirmed by Q-PCR. Thioglycollate-elicited macrophages were transfected with control or SRF siRNAs, as indicated, and the levels of Srf mRNA are shown, normalized to Gapdh expression. (C) Gene Spring representation of the gene expression profiles of control or SRF siRNA-transfected primary macrophages following the treatments indicated. The genes shown are those that were induced more than 3-fold after $6 \mathrm{~h}$ of zymosan treatment and were reduced by more than $33 \%$ with SRF knockdown at either 1 or $6 \mathrm{~h}$ of zymosan treatment. (D) Gene ontology (GO) analysis for biological process annotations of zymosan-induced genes $(6 \mathrm{~h})$ that were downregulated with SRF knockdown. (E) Highly enriched sequence motifs in the promoters of genes induced by zymosan $(6 \mathrm{~h})$ that were downregulated following SRF knockdown. 
immune responses based on Gene Ontology analysis using the Gene Spring software (Agilent) (Fig. 1D).

We next applied de novo computational motif discovery methods to search for enriched sequence motifs in the promoters ( $-500 \mathrm{bp}$ to $+500 \mathrm{bp}$ from the TSS) of the SRFdependent inflammatory response genes identified following zymosan treatment, as described above. Surprisingly, the sequence GAAACTGAAACC, but not a CArG-box, was identified as the most significantly enriched motif in genes exhibiting reduced induction at $6 \mathrm{~h}$ (Fig. 1E and Supplementary Table S2), but not at $1 \mathrm{~h}$ (data not shown). This motif is nearly identical to the consensus interferon-stimulated response element (ISRE) sequence (ggGAAAGTGAAACCa) (Levy and others 1988). The ISRE is recognized by IRFs and the type I IFN-inducible ISGF3 complex, which is formed by STAT1, STAT2, and IRF9 (Wesoly and others 2007). The identification of the ISRE element is unexpected because SRF has not been reported to biochemically or functionally interact with IRFs or any member of the ISGF3 complex.

\section{$S R F$ regulates the inflammatory response downstream of IFN- $\beta$ signaling}

Consistent with the identification of an ISRE sequence, most of the SRF-dependent, zymosan-induced genes identified by the microarray have previously been identified as ISGs. As the primary effectors of the IFN response, ISGs are indispensable for the antiviral response following viral infection (Takaoka and Yanai 2006). To confirm the time course and SRF dependence of known ISGs, we performed optimized Q-PCR assays to test ISG expression in siRNA treated samples after $0,1,3$, and $6 \mathrm{~h}$ of zymosan stimulation. Knockdown of SRF significantly inhibited the induction of ISGs such as Ifit2, Ifit3, Isg15, Ifit1, Irf7, Isg20, and Oas 2 after $6 \mathrm{~h}$ of zymosan treatment, but not after 1 or $3 \mathrm{~h}$ (Fig. 2A and Supplementary Fig. S1). This result confirms the observation from the microarray data that SRF is required for the full activation of these ISGs. Dectin-1 receptor and TLR2/ 6 ligands are not considered to be typical type I interferon response inducers. Consistent with this, interferon beta expression was only weakly induced ( $<3$-fold) 3 or $6 \mathrm{~h}$ following stimulation with Zymosan or FSL-1 (data not shown). We therefore examined the effect of SRF on the expression of ISGs after activation by polyI:C (recognized by TLR3) and LPS (recognized by TLR4). These experiments demonstrated that the loss of SRF also impaired ISG induction in response to TLR3 and TLR4 signaling (Fig. 2B, C), indicating that SRF has a general role in regulating the interferon response downstream of TLR activation.
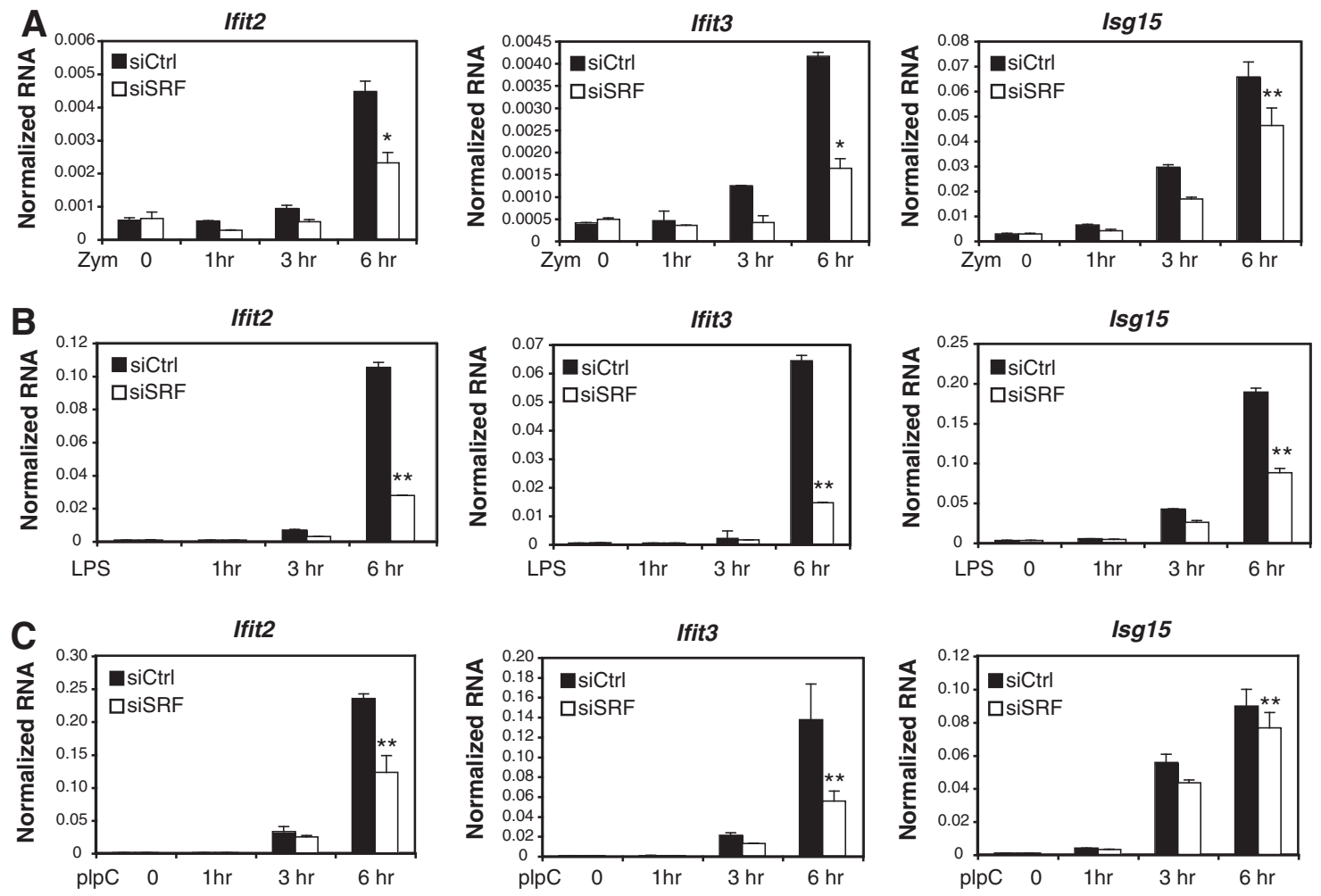

FIG. 2. Expression profiles of SRF-dependent interferon-stimulated genes (ISGs). (A, B, and C) mRNA expression profiles of representative SRF-dependent ISGs (Ifit2, Ifit3, Isg15) were analyzed by Q-PCR. Thioglycollate-elicited macrophages were transfected with control or SRF siRNAs, as indicated, and treated with (A) zymosan $(1 \mathrm{mg} / \mathrm{mL}),(B) \mathrm{LPS}(100 \mathrm{ng} / \mathrm{mL})$, and (C) polyI:C $(20 \mu \mathrm{g} / \mathrm{mL})$ for $0,1,3$, and $6 \mathrm{~h}$, respectively. Results shown are representative of 3 independent experiments. Statistical significance (denoted by asterisks) was determined by performing student's $t$-tests on the indicated mRNA levels after $6 \mathrm{~h}$ of zymosan stimulation with control or SRF-specific siRNA treatment in the 3 experiments ${ }^{*} P<0.05$ versus ctrl; ${ }^{*} P<0.01$ versus ctrl. Error bars represent the standard deviations of the duplicate PCR reactions used in the representative experiment shown. 


\section{SRF effect on ISG expression is specific for type I interferon-signaling}

To determine whether SRF functions upstream or downstream of interferon receptor signaling, we used IFN- $\beta$ and IFN- $\alpha$ to directly induce ISG expression in control and SRF knockdown cells. These experiments demonstrated that ISG expression was also impaired following SRF knockdown, suggesting that SRF acts downstream of type I interferon signaling to influence ISG responses (Fig. 3A, B). Interestingly, SRF-dependent ISG expression is specific for type I interferon-signaling because SRF siRNA-transfected cells treated with type II IFN- $\gamma$ did not exhibit defects in the induction of well-established IFN- $\gamma$ target genes, including Irf1, Oas2, and Stat1 (Fig. 3C).

\section{SRF knockdown antagonizes ISG expression independent of new protein synthesis and without affecting $m R N A$ stability}

To further examine SRF function in the interferon response, we tested whether the impairment of ISG expression in response to SRF knockdown was dependent on new protein synthesis. We hypothesized that SRF loss may affect intact ISG expression through either a defect of a secondary activator that is induced after IFN- $\beta$ stimulation or through the increased expression of negative regulators of the interferon pathway such as SIGIRR (single immunoglobulin IL1R-related molecule) or SOCS (Suppressor of cytokine signal) proteins (Coccia and others 2006). To exclude the possibility of the upregulation of a negative regulator following SRF knockdown, we pretreated control and SRF knockdown primary macrophages with cycloheximide to block new protein synthesis and then stimulated the cells with IFN- $\beta$. For the IFN- $\beta$-treated cells, SRF was required for an intact interferon response of Ifit2 both in the presence and absence of cycloheximide pretreatment indicating that SRF affected ISG transcription independent of new protein synthesis (Fig. 4A). The same result was seen for genes Ifit1 and Oas2 (data not shown). As a control for the effectiveness of the cycloheximide blockade, we also stimulated control and SRF knockdown primary macrophages with polyI:C after cycloheximide pretreatment. In this case, the induction of Ifit2 expression was impaired by cycloheximide pretreatment. This result is consistent with a block in the production of the type I interferons required for the secondary expression of ISGs and confirmed that new protein synthesis was indeed blocked in our experiment (Fig. 4B).

Experiments were also performed to determine whether the defects in the accumulation of ISG mRNA after SRF loss
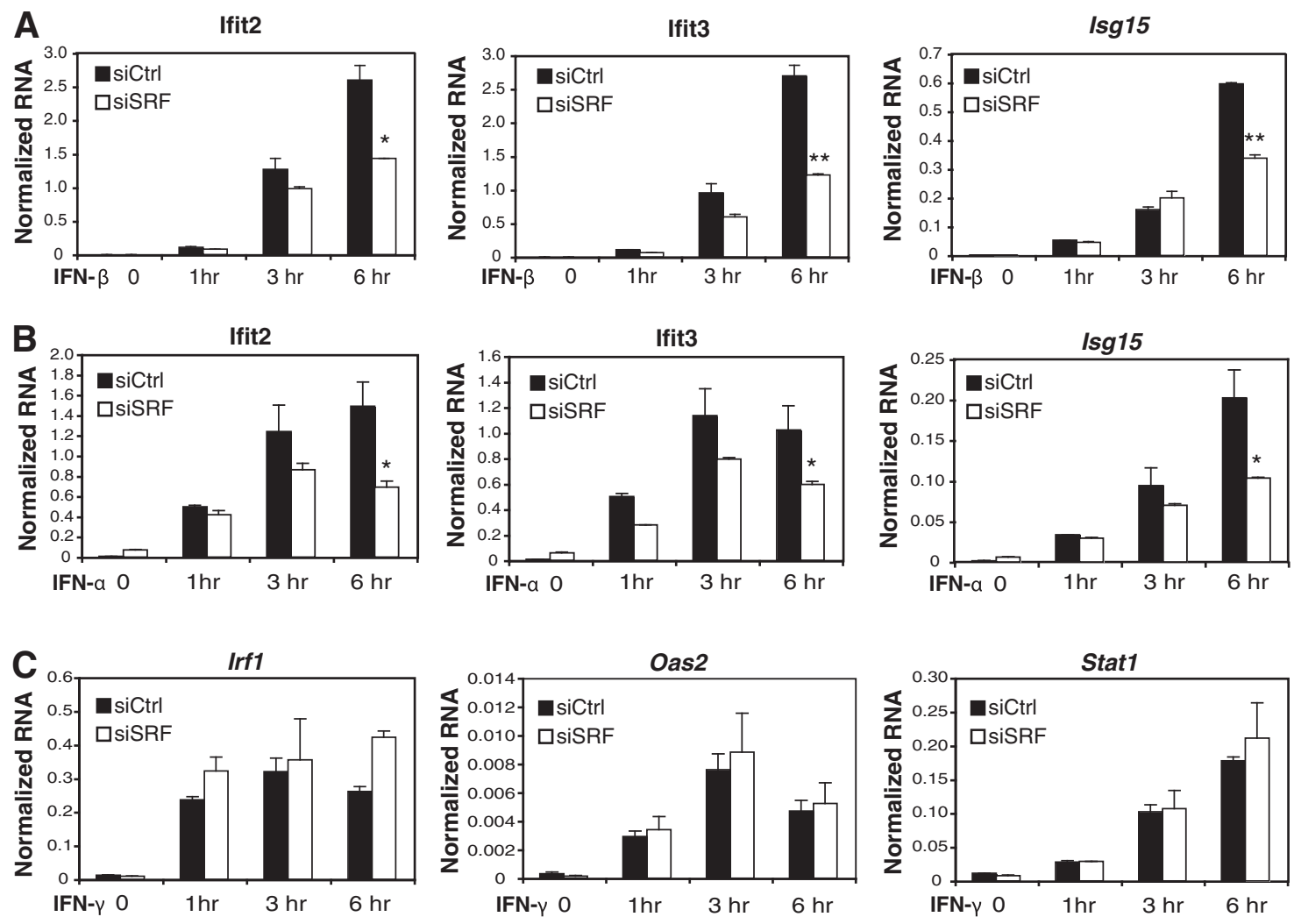

FIG. 3. SRF regulation of ISGs is specific for type I interferons. (A and B) Expression profiles of representative type I interferon-dependent genes (Ifit2, Ifit3, and Isg15) were analyzed by Q-PCR. Thioglycollate-elicited macrophages were transfected with control siRNA or siRNA targeted against SRF, as indicated, and left either untreated or treated with (A) IFN- $\beta$ (1000 Unit/mL) and (B) IFN- $\alpha$ (1000 Unit/mL) for 1, 3, or 6h. (C) Expression profiles of representative type II interferon-dependent genes (Irf1, Oas2, and Stat1) were analyzed by Q-PCR. siRNA transfections were carried out in primary macrophages as described in A and $\mathbf{B}$, but the cells were treated with IFN- $\gamma(20 \mu \mathrm{g} / \mathrm{mL})$ for $0,1,3$, or $6 \mathrm{~h}$. Results shown are representative of 3 independent experiments. Statistical significance (denoted by asterisks) was determined as described in Figure 2. ${ }^{*} P<0.05$ versus ctrl; ${ }^{* *} P<0.01$ versus ctrl. 

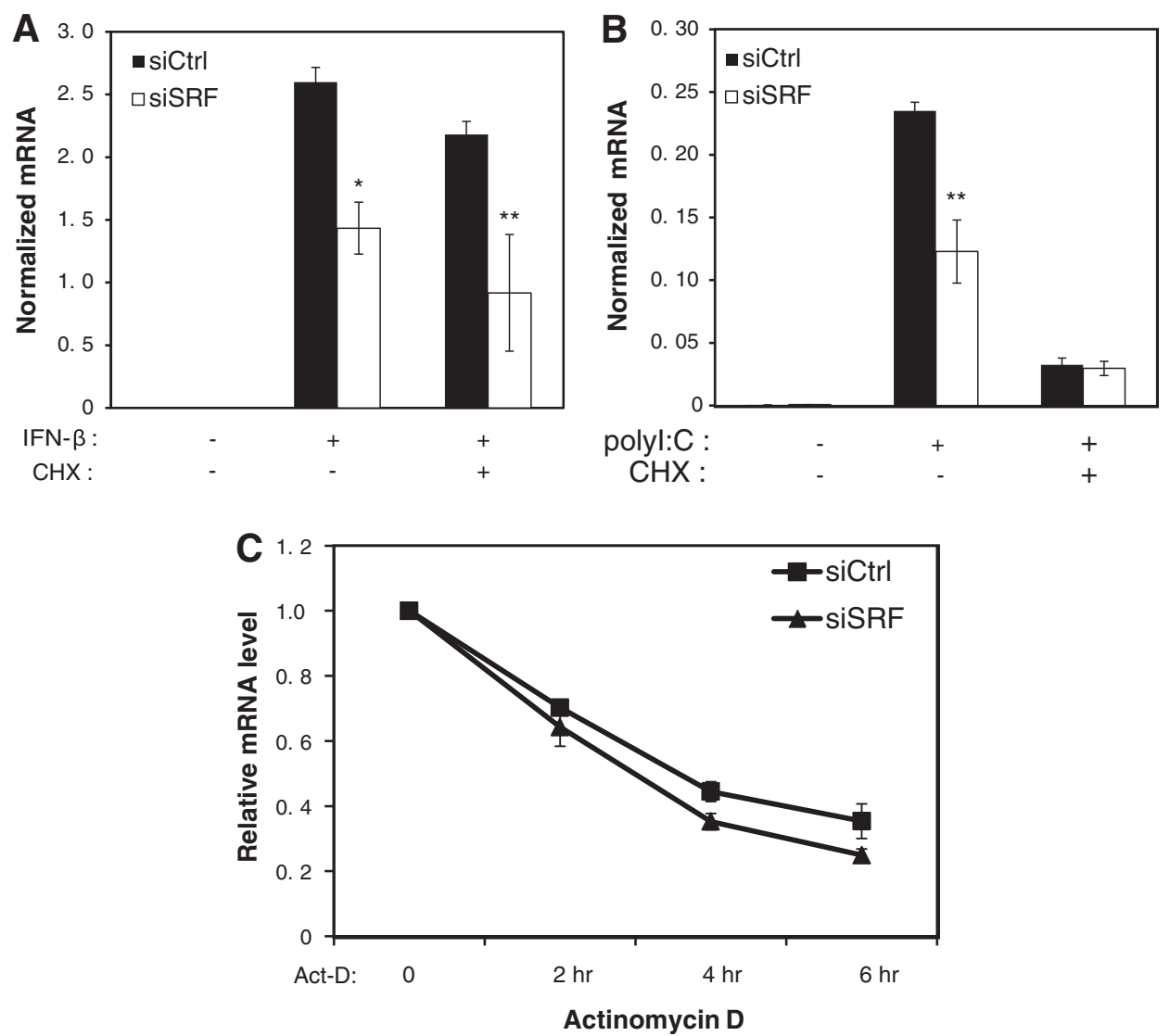

FIG. 4. SRF affects ISGs independent of protein synthesis and mRNA stability. (A and B) Ifit2 expression levels were analyzed by Q-PCR. Thioglycollate-elicited macrophages were transfected with control or SRF siRNAs, as indicated, and left either untreated or treated with (A) IFN- $\beta(1000$ Unit $/ \mathrm{mL})$ or (B) polyI:C $(20 \mu \mathrm{g} / \mathrm{mL})$ for $6 \mathrm{~h}$ in the absence or presence of cycloheximide $(10 \mu \mathrm{g} / \mathrm{mL})$. (C) Ifit3 expression levels were analyzed by Q-PCR in primary macrophages using the same siRNA knockdown procedure as described in A and B. Cells were untreated or treated with IFN- $\beta$ (1000 Unit/mL) for $6 \mathrm{~h}$, Act-D $(1 \mu \mathrm{g} / \mathrm{mL})$ was then added, and cells were harvested $0,2,4$, and $6 \mathrm{~h}$ after Act-D treatment, as indicated. Results are expressed as the mRNA levels observed relative to the appropriate control or SRF siRNA-transfected samples induced with IFN- $\beta$ without subsequent Act-D treatment $(0 \mathrm{~h})$. Results shown are the average relative expressions observed in 3 independent experiments.

were due to decreased transcription of the mRNA or decreased mRNA stability. To distinguish these possibilities, control and SRF-specific siRNA knockdown macrophages were first treated with IFN- $\beta(1000 \mathrm{U} / \mathrm{mL})$ for $6 \mathrm{~h}$ to induce an initial wave of transcription and then treated with the transcription inhibitor, Actinomycin D (Act-D), to block transcription. RNA was harvested at $0,2,4$, and $6 \mathrm{~h}$ after Act-D treatment, respectively, and Ifit3 expression was analyzed. Our results indicated that there were no significant differences in the Ifit3 mRNA levels in the control or SRFspecific siRNA-transfected samples following Act-D treatment relative to the induced Ifit3 expression levels (Fig. 4C). These results suggest that SRF knockdown does not cause decreases in ISG expression through increased mRNA turnover.

\section{SRF does not affect the IFN-JAK-STAT-ISGF3 pathway}

One possible mechanistic explanation for the observed defect in ISG activation after SRF loss could be the negative regulation of the JAK-STAT-ISGF3 signaling pathway. The effect of SRF expression on type I interferon signal transduction was tested by examining the expression, phosphorylation, and nuclear accumulation of STAT1 and STAT2 after IFN- $\beta$ stimulation. Western blots performed on macrophage whole cell extracts showed that protein levels of STAT1 and STAT2 are somehow increased with SRF knockdown in untreated conditions and are not changed with or without SRF knockdown after IFN- $\beta$ stimulation. Moreover, immunoblotting with anti-phospho-STAT1/2 antibodies indicated that the IFN- $\beta$-dependent activation of STAT1 and STAT2 was also not inhibited by SRF loss (Fig. 5A). The subcellular distributions of STAT1 and STAT2, and the phosphorylated STAT proteins, were also examined and showed no dependence on the presence of SRF, both at an early time point (30 min, Fig. 5B) and a late time point $(18 \mathrm{~h}$, data not shown). These results indicate that IFN- $\beta$-responsive signal transduction from the cytoplasm to the nucleus remains intact after SRF loss, suggesting that SRF is required in another intra-nuclear step in ISG induction.

To identify whether SRF directly regulates ISG expression, we checked SRF binding sites on a genome-wide scale using 
A

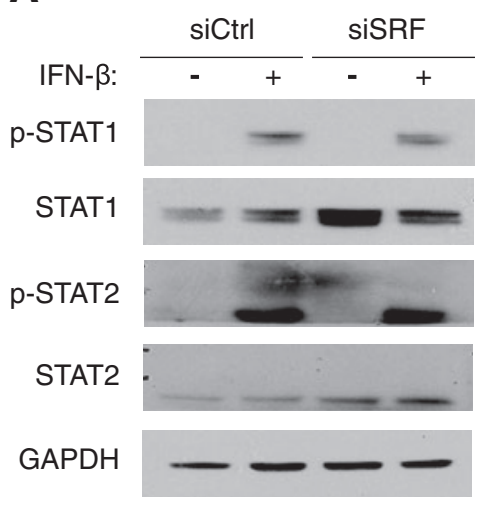

B

IFN- $\beta$ :

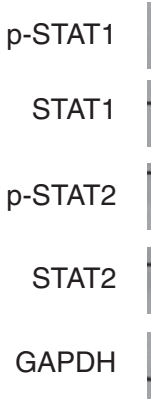

lamin

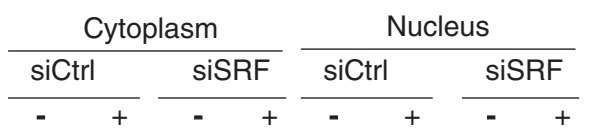
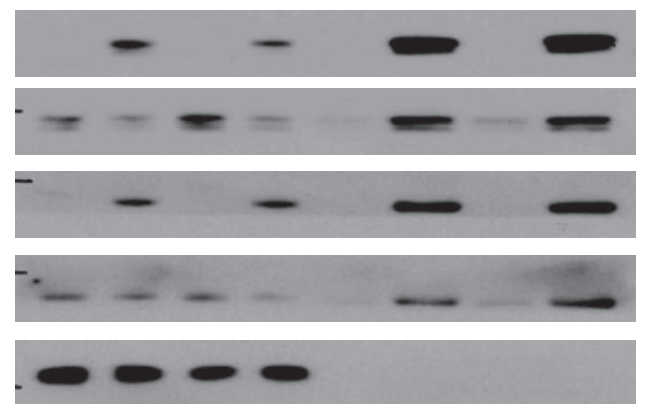

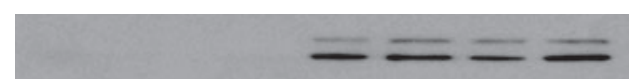

FIG. 5. SRF affects ISGs without affecting the expression, phosphorylation, and nuclear translocation of ISGF3 signaling molecules. (A) Western blots showing consistent levels of the indicated proteins with or without SRF knockdown, before or after treatment with $1000 \mathrm{Unit} / \mathrm{mL}$ of IFN- $\beta$ for $30 \mathrm{~min}$. Lysates were probed with anti-GAPDH antibodies as a loading control. (B) Nuclear and cytoplasmic extracts from primary macrophages transfected with control or SRF siRNAs and treated with IFN- $\beta$ for 0 or $30 \mathrm{~min}$, as indicated, were immunoblotted for the indicated proteins. Extracts were probed with antilamin and anti-GAPDH antibodies to show the integrity of the nuclear and cytoplasmic extracts, respectively, and to serve as loading controls. Results shown are representative of 3 independent experiments.

chromatin immunoprecipitation coupled to deep sequencing (ChIP-Seq) in primary macrophages. Our ChIP-Seq analysis of SRF resulted in the identification of more than 1,000 binding sites (Sullivan and others 2010), including binding sites in the well-known SRF target genes $c f o s$ (Treisman 1986) and vinculin $(\mathrm{Vcl})$ (Cen and others 2003). However, none of the SRF-dependent ISG promoters showed occupancy by SRF (data not shown), consistent with an indirect effect of SRF on the transcription of ISGs.

\section{Discussion}

While essential roles of SRF in embryogenesis, neuronal development, smooth muscle differentiation, and cardiac cell differentiation have been extensively documented (Miano 2003), much less is known concerning the functions of SRF in the immune system. Studies in lymphocytes have demonstrated that T cell-specific deletion of Srf results in a severe block in thymocyte development at the transition from CD4/ CD8 double to single positive stage, while B cell-specific Srf deletion leads to a complete loss of marginal zone B cells and a marked reduction of the CD5+B cell subset (Fleige and others 2007). These results indicate essential and distinct functions of SRF in the differentiation of T and B cells. Additional evidence relating SRF to immune system function was provided by a study demonstrating that LPS activation of tissue factor and TNF- $\alpha$ expression in monocytic cells involved SRF-dependent induction of egr-1 (Guha and others 2001).

Here, we provide evidence that SRF is required for optimal transcriptional responses of primary macrophages to type I interferons. Transcriptional profiling of zymosantreated macrophages led to the identification of a CArG-box motif as being significantly enriched in upregulated promoters. Transcriptome analysis of SRF-deficient cells also indicated that SRF is required for the induction of both early $(1 \mathrm{~h})$ and late $(6 \mathrm{~h})$ subsets of zymosan-inducible genes. Unexpectedly, de novo motif analysis of late inducible gene promoters dependent on SRF for induction revealed an ISRE sequence as the most highly enriched transcription factor binding site, suggesting a role of SRF in regulating interferon-dependent gene expression. These results were confirmed and extended by the demonstration of a requirement for SRF for full induction of numerous ISGs in response to zymosan, polyI:C, and LPS. Based on our findings, it is tempting to speculate that SRF function is important for immune responses against viral infection, since the virus-triggered IFN- $\beta$ pathway is critical for host survival.

Molecular analysis indicates that the impairment of ISG induction in SRF-deficient macrophages in response to TLR activation primarily results from a defect in the ability of induced type I interferons to mediate secondary gene expression. Intriguingly, this effect is limited to the type I interferon pathway, as type II interferon-induced target gene activation was not affected by SRF knockdown. The underlying mechanism responsible for this defect remains to be established. Our studies showed that phosphorylation and nuclear translocation of STAT1/2 in response to IFN- $\beta$ normally occurs in SRF-deficient macrophages. We also excluded the possibility that loss of SRF could affect the persistence of ISGF3 in the nucleus, because the amount of phosphorylated STAT1/2 in the nucleus was not changed in the absence of SRF up to $18 \mathrm{~h}$ following IFN- $\beta$ treatment. Even though SRF ChIP-Seq did not reveal direct SRF binding to these ISRE-containing genes, it is possible that SRF could act as a non-DNA binding coactivator to promote transcription complex formation with STATs. Alternatively, SRF may be required for proper expression of a co-activator that is required for persistent gene activation. Further studies are needed to investigate the complete mechanism of how SRF is involved in the regulation of ISG expression.

We previously demonstrated that SRF primarily regulates cytoskeleton-dependent functions in macrophages under basal conditions (Sullivan and others 2010). Here, our studies suggest that SRF regulates an inflammatory gene program, 
in addition to the immediate early genes and cytoskeletal genes that have already been described. These findings suggest that SRF is involved in the establishment of an antiviral state in macrophages, and thus is an important factor for regulating innate immunity.

\section{Acknowledgments}

We are grateful to Dr. Christopher Benner and Dr. Michael David for helpful discussion and suggestions. We thank the UCSD Biogem Core facility for labeling and hybridization of the expression microarray samples. We also thank Lynn Bautista for her assistance with preparation of the figures. This work was supported by NIH grants CA52599 and DK063491.

\section{Author Disclosure Statement}

No competing financial interests exist.

\section{References}

Akira S, Takeda K. 2004. Toll-like receptor signalling. Nat Rev Immunol 4(7):499-511.

Alberti S, Krause SM, Kretz O, Philippar U, Lemberger T, Casanova E, Wiebel FF, Schwarz H, Frotscher M, Schutz G, Nordheim A. 2005. Neuronal migration in the murine rostral migratory stream requires serum response factor. Proc Natl Acad Sci U S A 102(17):6148-6153.

Barton GM, Medzhitov R. 2003. Toll-like receptor signaling pathways. Science 300(5625):1524-1525.

Cen B, Selvaraj A, Burgess RC, Hitzler JK, Ma Z, Morris SW, Prywes R. 2003. Megakaryoblastic leukemia 1, a potent transcriptional coactivator for serum response factor (SRF), is required for serum induction of SRF target genes. Mol Cell Biol 23(18):6597-6608.

Chai J, Tarnawski AS. 2002. Serum response factor: discovery, biochemistry, biological roles and implications for tissue injury healing. J Physiol Pharmacol 53(2):147-157.

Coccia EM, Uze G, Pellegrini S. 2006. Negative regulation of type I interferon signaling: facts and mechanisms. Cell Mol Biol (Noisy-le-grand) 52(1):77-87.

Fleige A, Alberti S, Grobe L, Frischmann U, Geffers R, Muller W, Nordheim A, Schippers A. 2007. Serum response factor contributes selectively to lymphocyte development. J Biol Chem 282(33):24320-24328.

Gale M, Jr., Foy EM. 2005. Evasion of intracellular host defence by hepatitis $C$ virus. Nature 436(7053):939-945.

Ghisletti S, Huang W, Ogawa S, Pascual G, Lin ME, Willson TM, Rosenfeld MG, Glass CK. 2007. Parallel SUMOylationdependent pathways mediate gene- and signal-specific transrepression by LXRs and PPARgamma. Mol Cell 25(1):57-70.

Goodridge HS, Harnett MM. 2005. Introduction to immune cell signalling. Parasitology 130 (Suppl):S3-S9.

Guha M, O'Connell MA, Pawlinski R, Hollis A, McGovern P, Yan SF, Stern D, Mackman N. 2001. Lipopolysaccharide activation of the MEK-ERK1/2 pathway in human monocytic cells mediates tissue factor and tumor necrosis factor alpha expression by inducing Elk-1 phosphorylation and Egr-1 expression. Blood 98(5):1429-1439.

Heintzman ND, Hon GC, Hawkins RD, Kheradpour P, Stark A, Harp LF, Ye Z, Lee LK, Stuart RK, Ching CW, Ching KA, Antosiewicz-Bourget JE, Liu H, Zhang X, Green RD, Lobanenkov VV, Stewart R, Thomson JA, Crawford GE, Kellis M, Ren B. 2009. Histone modifications at human enhancers reflect global cell-type-specific gene expression. Nature 459(7243): 108-112.

Heinz S, Benner C, Spann N, Bertolino E, Lin YC, Laslo P, Cheng JX, Murre C, Singh H, Glass CK. 2010. Simple combinations of lineage-determining transcription factors prime cis-regulatory elements required for macrophage and B cell identities. Mol Cell 38(4):576-589.

Honda K, Taniguchi T. 2006. IRFs: master regulators of signalling by Toll-like receptors and cytosolic pattern-recognition receptors. Nat Rev Immunol 6(9):644-658.

Impey S, Fong AL, Wang Y, Cardinaux JR, Fass DM, Obrietan K, Wayman GA, Storm DR, Soderling TR, Goodman RH. 2002. Phosphorylation of CBP mediates transcriptional activation by neural activity and CaM kinase IV. Neuron 34(2):235-244. Karumuthil-Melethil S, Perez N, Li R, Vasu C. 2008. Induction of innate immune response through TLR2 and dectin 1 prevents type 1 diabetes. J Immunol 181(12):8323-8334.

Lahoute C, Sotiropoulos A, Favier M, Guillet-Deniau I, Charvet C, Ferry A, Butler-Browne G, Metzger D, Tuil D, Daegelen D. 2008. Premature aging in skeletal muscle lacking serum response factor. PLoS One 3(12):e3910.

Lehtonen A, Matikainen S, Julkunen I. 1997. Interferons upregulate STAT1, STAT2, and IRF family transcription factor gene expression in human peripheral blood mononuclear cells and macrophages. J Immunol 159(2):794-803.

Levy DE, Kessler DS, Pine R, Reich N, Darnell JE, Jr. 1988. Interferon-induced nuclear factors that bind a shared promoter element correlate with positive and negative transcriptional control. Genes Dev 2(4):383-393.

Li Q, Verma IM. 2002. NF-kappaB regulation in the immune system. Nat Rev Immunol 2(10):725-734.

Livak KJ, Schmittgen TD. 2001. Analysis of relative gene expression data using real-time quantitative $\mathrm{PCR}$ and the 2(-Delta Delta C(T)) Method. Methods 25(4):402-408.

Miano JM. 2003. Serum response factor: toggling between disparate programs of gene expression. J Mol Cell Cardiol 35(6):577-593.

Miano JM, Long X, Fujiwara K. 2007. Serum response factor: master regulator of the actin cytoskeleton and contractile apparatus. Am J Physiol Cell Physiol 292(1):C70-C81.

Miano JM, Ramanan N, Georger MA, de Mesy Bentley KL, Emerson RL, Balza RO, Jr., Xiao Q, Weiler H, Ginty DD, Misra RP. 2004. Restricted inactivation of serum response factor to the cardiovascular system. Proc Natl Acad Sci U S A 101(49):17132-17137.

Panne D, Maniatis T, Harrison SC. 2004. Crystal structure of ATF-2/c-Jun and IRF-3 bound to the interferon-beta enhancer. EMBO J 23(22):4384-4393.

Schratt G, Philippar U, Berger J, Schwarz H, Heidenreich O, Nordheim A. 2002. Serum response factor is crucial for actin cytoskeletal organization and focal adhesion assembly in embryonic stem cells. J Cell Biol 156(4):737-750.

Sullivan AL, Benner C, Heinz S, Huang W, Xie L, Miano JM, Glass CK. 2010. Serum response factor utilizes distinct promoter- and enhancer-based mechanisms to regulate cytoskeletal gene expression in macrophages. Mol Cell Biol 31(4):861-875.

Sun K, Battle MA, Misra RP, Duncan SA. 2009. Hepatocyte expression of serum response factor is essential for liver function, hepatocyte proliferation and survival, and postnatal body growth in mice. Hepatology 49(5):1645-1654.

Sun Q, Chen G, Streb JW, Long X, Yang Y, Stoeckert CJ, Jr., Miano JM. 2006. Defining the mammalian CArGome. Genome Res 16(2):197-207.

Takaoka A, Yanai H. 2006. Interferon signalling network in innate defence. Cell Microbiol 8(6):907-922. 
Treisman R. 1986. Identification of a protein-binding site that mediates transcriptional response of the c-fos gene to serum factors. Cell 46(4):567-574.

Wesoly J, Szweykowska-Kulinska Z, Bluyssen HA. 2007. STAT activation and differential complex formation dictate selectivity of interferon responses. Acta Biochim Pol 54(1): 27-38.

Yadav M, Schorey JS. 2006. The beta-glucan receptor dectin-1 functions together with TLR2 to mediate macrophage activation by mycobacteria. Blood 108(9):3168-3175.
Address correspondence to: Dr. Christopher K. Glass

Department of Cellular and Molecular Medicine University of California, San Diego 9500 Gilman Drive La Jolla, CA 92093-0651

E-mail: ckg@ucsd.edu

Received 11 March 2013/Accepted 13 March 2013 